\title{
Clec14a genetically interacts with Etv2 and Vegf signaling during vasculogenesis and angiogenesis in zebrafish
}

\author{
Karolina Pociute ${ }^{1,2}$, Jennifer A. Schumacher ${ }^{1,3}$ and Saulius Sumanas ${ }^{1,3^{*}}$
}

\begin{abstract}
Background: C-lectin family 14 Member A (Clec14a) is a transmembrane protein specifically expressed in vascular endothelial cells during embryogenesis. Previous in vitro and in vivo studies have provided conflicting data regarding Clec14a role in promoting or inhibiting angiogenesis, therefore its functional role in vascular development remains poorly understood.

Results: Here we have generated a novel clec14a mutant allele in zebrafish embryos using TALEN genome editing. clec14a mutant embryos exhibit partial defects and delay in the sprouting of intersegmental vessels. These defects in angiogenesis are greatly increased upon the knockdown of a structurally related C1qr protein. Furthermore, a partial knockdown of an ETS transcription factor Etv2 results in a synergistic effect with the clec14a mutation and inhibits expression of early vascular markers in endothelial progenitor cells, arguing that clec14a is involved in promoting vasculogenesis. In addition, Clec14a genetically interacts with Vegfa signaling. A partial knockdown of Vegfaa function in the clec14a mutant background resulted in a synergistic inhibition of intersegmental vessel sprouting.
\end{abstract}

Conclusions: These results argue that clec14a is involved in both vasculogenesis and angiogenesis, and suggest that Clec14a genetically interacts with Etv2 and Vegf signaling during vascular development in zebrafish embryos.

Keywords: Clec14, Zebrafish, Angiogenesis, Vasculogenesis, Vascular endothelial, Vegf, etv2, Etsrp

\section{Background}

New blood vessels form by two distinct mechanisms, vasculogenesis and angiogenesis. Vasculogenesis involves formation of new blood vessels through differentiation of vascular endothelial cells de novo, while angiogenesis involves growth of new vasculature through sprouting from the existing blood vessels [1]. Despite a significant progress in identifying signaling pathways that regulate vascular development, the molecular mechanisms that regulate vasculogenesis and angiogenesis are still only partially understood.

C-type lectin family 14 member A (Clec14a, also known as $\mathrm{C} 1 \mathrm{qrl}, \mathrm{Crl})$ is a transmembrane protein which contains a C-type lectin and EGF-like domains [2]. Its protein

\footnotetext{
*Correspondence: saulius.sumanas@cchmc.org

'Division of Developmental Biology, Cincinnati Children's Hospital Medical

Center, 3333 Burnet Ave, Cincinnati, OH 45229, USA

${ }^{3}$ Department of Pediatrics, University of Cincinnati College of Medicine, 3333

Burnet Ave, Cincinnati, $\mathrm{OH}$ 45229, USA

Full list of author information is available at the end of the article
}

sequence is highly conserved between multiple vertebrates including zebrafish, mouse and humans. We have previously described its expression in vascular endothelial cells in zebrafish embryos [3]. Expression of clec14a was greatly downregulated in cloche / npas $4 l$ mutants, deficient in hematopoietic and vascular development. Similar to zebrafish, Clec14a is specifically expressed in vascular endothelial cells in mouse embryos and human tissues, and its expression is greatly upregulated during tumor angiogenesis [2, 4]. In vitro studies have demonstrated that CLEC14A promotes filopodia formation, cell migration and tubulogenesis [2, 4]. In zebrafish, it has been reported that Clec14a functions redundantly with a related protein C1qr / Cd93 in promoting angiogenesis [5]. Double clec14a and c1qr mutant embryos showed greatly inhibited angiogenesis and reduced cadherin 5 (cdh5) expression, which could be rescued by synthetic $c d h 5$ mRNA injection [5]. In contrast, mouse Clec14a mutants displayed increased angiogenesis and lymphangiogenesis, accompanied by an increase in hemorrhages and vessel

(c) The Author(s). 2019 Open Access This article is distributed under the terms of the Creative Commons Attribution 4.0 International License (http://creativecommons.org/licenses/by/4.0/), which permits unrestricted use, distribution, and 
dilations [6]. Clec14a deficiency resulted in reduced endothelial expression of Vascular Endothelial Growth Factor Receptor 3 (Vegfr3), while expression of Vegfr2 was increased. In addition, Clec14a was shown to physically interact with Vegfr3 [6].

While all previous studies point to the role of Clec14a in regulating angiogenesis, it is currently unclear why the Clec14a knockout in mouse embryos results in increased angiogenesis, while the zebrafish clec14a mutants show reduced angiogenesis, similar to the CLEC14A knockdown in cell culture. Furthermore, it is currently unknown if Clec14a plays any role in vasculogenesis, in addition to its previously reported role in angiogenesis. To address these questions, we generated a novel zebrafish clec14a mutant allele using transcription activator-like effector nucleases (TALEN)- mediated genome editing. Our results show that clec14a mutants display subtle defects in angiogenic sprouting which are greatly increased upon functional inhibition of a related C1qr protein. We demonstrate that clec14a genetically interacts with ETS transcription factor etv 2 during vasculogenesis, demonstrating its novel role in promoting differentiation of vascular endothelial progenitors. We also show a synergistic genetic interaction between clec14a and Vegf signaling.

\section{Results}

To analyze the function of zebrafish clec14a during vascular development, we generated clec14a mutant allele using TALEN genome editing [7]. The clec14a $a^{c i 15}$ mutant allele carries a $10 \mathrm{bp}$ deletion and is predicted to result in a frameshift and premature stop codon early in the open reading frame at amino acid position 44 (Fig. 1a, b). DNA sequencing of clec14a coding sequence amplified by PCR from cDNA obtained from clec14a mutant embryos at 24 hpf confirmed the presence of expected $10 \mathrm{bp}$ deletion (Fig. 1c, d). No other splice variants or additional PCR bands were identified in clec14a cDNA of clec14a mutant embryos (data not shown). Expression of clec14a was greatly reduced in clec14a homozygous mutant embryos as analyzed by in situ hybridization (ISH) (Fig. 1c, d). These data argue that the level of clec14a mRNA is greatly reduced in clec14a mutants, and the remaining transcript does not code for a functional protein, suggesting that the mutation allele is null or close to null. Nevertheless, homozygous clec14a mutant embryos were morphologically normal, did not show any obvious defects and were viable as adults (data not shown).

A previous study suggested that Clec14a functions redundantly with a related protein C1qr [5]. Recent studies have shown that expression of functional homologs is often upregulated in genetic mutants as a part of a compensatory mechanism [8]. Indeed, c1qr expression was increased by 2.9-fold ( \pm 0.7 SEM) in clec14a-/- mutant embryos at 24 hpf by qPCR analysis compared to wild-type embryos ( $p=$
0.03 , Student's t-test). To test if $c 1 q r$ compensates for the loss of clec14a function, we designed and injected a translation-blocking morpholino $(\mathrm{MO})$ against $\mathrm{C} 1 \mathrm{qr}$ protein in either wild-type or clec14a-/- embryos crossed into vascular endothelial specific kdrl: GFP transgenic background. Phenotypic analysis revealed that about $20 \%$ of clec14a-/- mutants and $12.5 \%$ of c1qr MO injected embryos showed reduced or delayed intersegmental vessel (ISV) sprouting at $28 \mathrm{hpf}$. This percentage was slightly higher (26.5\%) in double knockdown c1qr MO; clec14a-/embryos, which was not a statistically significant difference (Fig. 2a-d, m). Sprouting defects including truncated or missing ISVs and abnormal ISV connections persisted in a significant fraction (16-33\%) of clec14a-/- or c1qr MO injected embryos at 48 and 72 hpf (Fig. 2e-n). In contrast, the majority of double knockdown c1qr MO; clec14a-/embryos (60-64\%) displayed abnormal ISV connections, mispatterned ISVs, partial ISV sprouts and defects in the formation of the dorsal longitudinal anastomotic vessel (DLAV) (Fig. 2h, l-n). Overall, the ISV sprouting defects observed in c1qr MO; clec14a-/- embryos were similar to the previously reported defects in c1qr; clec14a double mutants [5], arguing that the MO specifically inhibits $\mathrm{C} 1 \mathrm{qr}$ function. To confirm that c1qr $M O$ effectively inhibits protein expression, we designed a GFP reporter by fusing 939 bp of clqr genomic sequence immediately upstream of the translation-initiating ATG codon to the GFP reporter and SV40 polyA sequence. $11 \%$ of embryos injected with this construct showed GFP expression in multiple cells in the trunk and tail region while none of the embryos co-injected with the GFP reporter and c1qr MO showed such expression (Additional file 1: Figure S1). This argues that $c 1 q r$ MO can effectively inhibit GFP reporter expression. Injection of a control $\mathrm{MO}$ or a 5-base mismatch $\mathrm{MO}$ into clec14a mutants did not affect the percentage of embryos with ISV defects, further arguing for the specificity of the observed phenotype (Additional file 1: Figures S2 and S3). Thus, C1qr and Clec14a function partially redundantly during angiogenic sprouting.

We then analyzed expression of vascular markers in clec14a-/- mutants, c1qr MO-injected embryos and double clec14a-/-; c1qr MO embryos using in situ hybridization (ISH). The majority of clec14a-/- or clqr MO embryos displayed normal expression of Vegfr2 homolog $k d r l$, ETS transcription factor flila and VE-cadherin / cdh5, while a fraction of these embryos (13-34\%) exhibited inhibition of ISV sprouting (Fig. 3a-c, e-g, i-k, u, and data not shown). The percentage of affected embryos correlated closely to the percentage of embryos showing ISV defects based on $k d r l$ : GFP fluorescence analysis. In contrast, double clqrMO; clec14a-/- embryos showed strong reduction in ISV sprouting (Fig. 3d, h, l, u). Expression of the arterial marker $c l d n 5 b$ in the dorsal aorta (DA) and venous marker flt4 in the posterior cardinal vein 

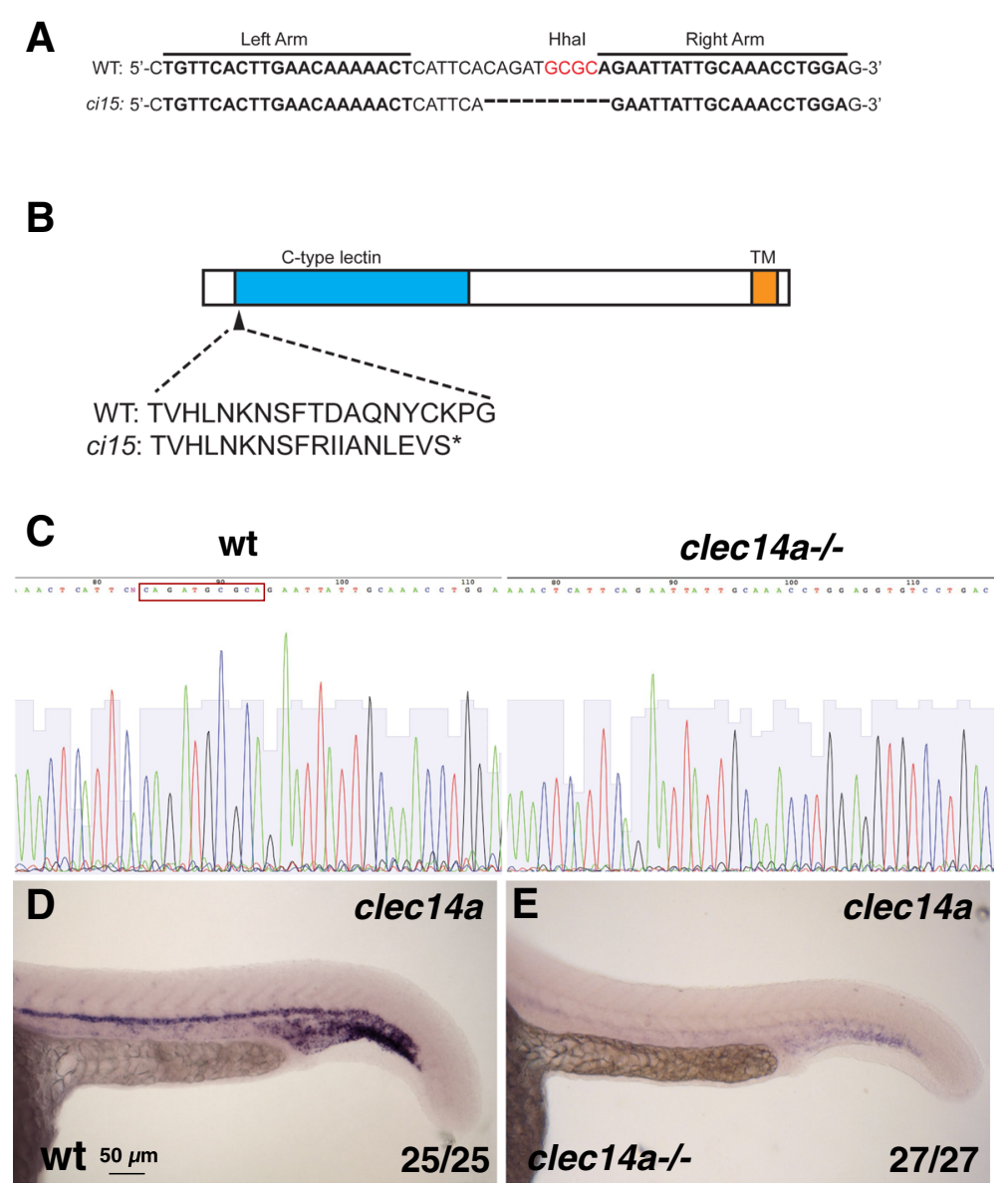

Fig. 1 Generation of clec14a mutants by TALEN-mediated genome editing. a Wild-type and clec14a ${ }^{\text {ci15 }}$ mutant sequence showing the binding regions for TALE nucleases and the 10 bp deletion in the clec $14 a$ mutants which includes the deletion of Hhal restriction site used for genotyping. $\mathbf{b} \mathrm{A}$ diagram of Clec14a protein sequence which includes C-type lectin and transmembrane (TM) domains. clec $14 a^{\text {cil5 }}$ mutation is predicted to cause a frameshift early in the protein coding sequence starting at the amino acid 44 and would lead to a premature stop codon. $\mathbf{c}, \mathbf{d}$ DNA sequencing chromatogram shows a 10 bp deletion (boxed-in wild-type sequence) in the cDNA of clec14a mutants. $\mathbf{d}$, e In situ hybridization analysis for clec14a expression in wt embryos (d) and clec14a mutants (e) at $24 \mathrm{hpf}$. Note a significant reduction of clec 14a expression in clec14a mutant embryos. The experiment has been replicated twice; the combined number of embryos analyzed and showing the phenotype is shown in the lower right corner

(PCV) was not significantly affected in clec14a-/-, c1qrMO or double knockdown embryos, suggesting that arteriovenous patterning was not affected while ISV sprouting was partially inhibited, similar to the results obtained with the other markers (Fig. 3m-u).

To test if clec14a may participate in early vasculogenesis, we analyzed its genetic interaction with the ETS transcription factor Etv2 / Etsrp, a key regulator of early vasculogenesis $[9,10]$. Injection of low doses of 0.125 or $0.25 \mathrm{ng}$ per embryo of the previously validated etv2 morpholino [9] resulted in partial defects in vasculogenesis and angiogenic sprouting. ISV sprouting defects at $48 \mathrm{hpf}$ were more severe in clec14a-/-; kdrl: GFP embryos injected with 0.25 ng of etv2 MO compared to wild-type kdrl: GFP embryos injected with the same dose of etv2 MO (Fig. 4a-d). The number of partial ISVs, absent ISVs and abnormal ISV connections per embryo was significantly increased in clec14a
-/-; etv2 MO embryos compared with wild-type embryos injected with etv2 $\mathrm{MO}$ (Fig. $4 \mathrm{~m}$-o; because the majority of clec14a-/- embryos do not show ISV defects, they were not included in this analysis). While $31 \%$ of wild-type etv2 MO injected embryos had normal axial blood circulation at $48 \mathrm{hpf}$, only $3 \%$ of clec14a-/-; etv $2 \mathrm{MO}$ embryos had normal circulation, while the rest showed partially or completely inhibited axial circulation (Fig. 4p, Additional file 2: Movie S1 and Additional file 3: Movie S2). To test if clec14a-/- contributed to early vasculogenesis, we analyzed $k d r l$ and $c d h 5$ expression in vascular endothelial progenitors at the 15-16-somite stages. There was no apparent difference between the majority of clec14a-/embryos and wild-type controls while a fraction of clec14a mutant embryos showed a slight reduction in $k d r l$ and cdh5 expression (Fig. 4e, f, i, j, q). Wild-type embryos injected with a low $0.125 \mathrm{ng}$ dose of etv2 MO showed 


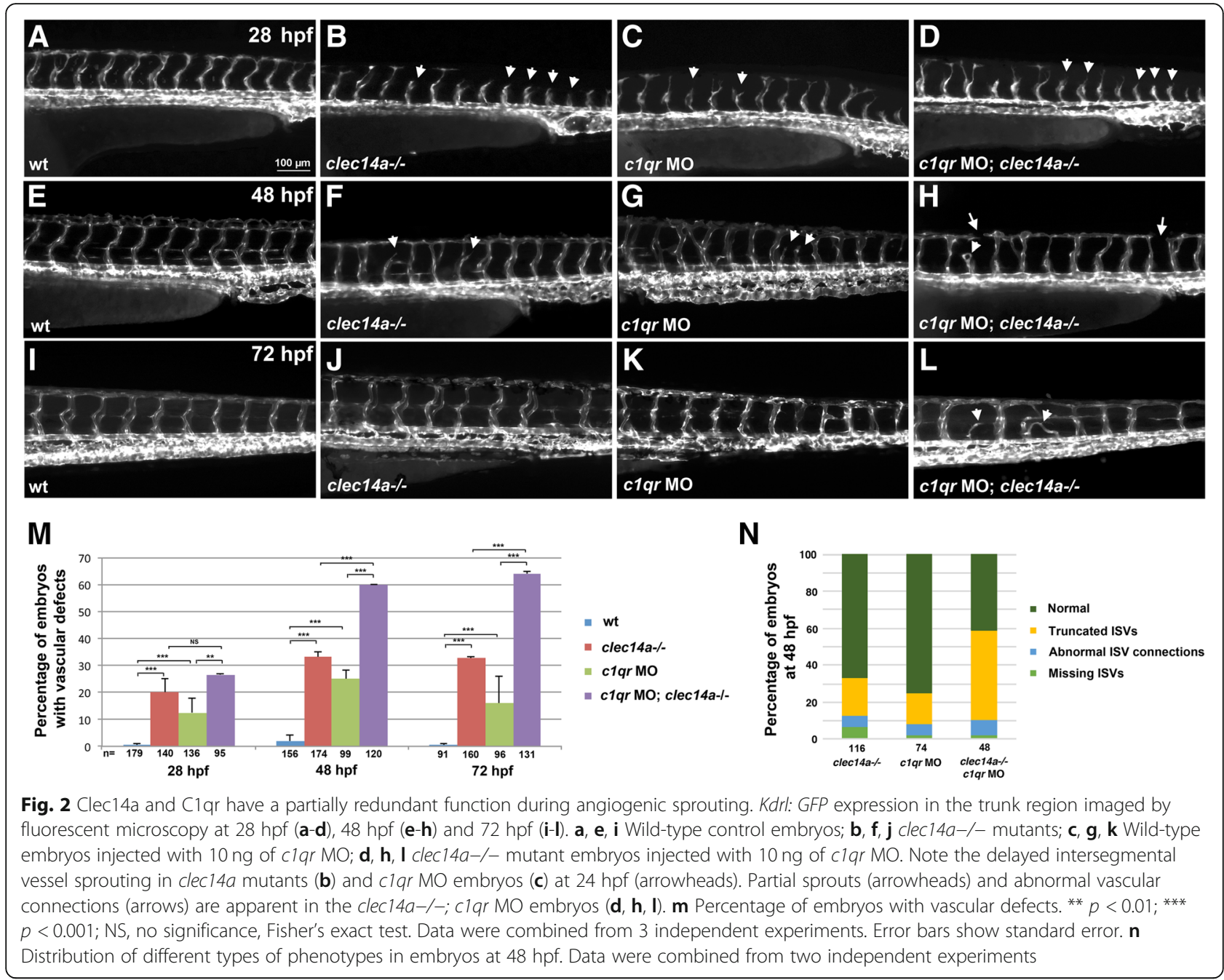

significant inhibition in $k d r l$ and $c d h 5$ expression (Fig. $4 \mathrm{~g}$, $\mathrm{k}, \mathrm{q})$. Injection of the same dose of etv2 MO into clec14a $-/-$ embryos resulted in a much stronger inhibition in expression of these markers. In most embryos, very few cells with weak $k d r l$ and $c d h 5$ expression were present (Fig. 4h, $\mathrm{l}, \mathrm{q})$. Because the phenotype of this severity was observed only in the double etv2 MO; clec14a-/- embryos, this indicates a synergistic effect between etv2 MO knockdown and clec14a mutation. These results suggest that clec14a contributes to vasculogenesis and functions during the specification and differentiation of vascular endothelial progenitors.

We then tested genetic interaction between clec14a and Vegf signaling. As analyzed by $k d r l$ expression at 24 hpf, low dose injection of the previously validated vegfaa MO [11] resulted in a partial reduction of ISV sprouting while axial vessel development appeared unaffected (Fig. 5c). Injection of vegfaa MO in clec14a-/- mutants resulted in a much stronger effect. The majority of embryos had no ISV sprouting at 24 hpf (Fig. 5a-d, i). More severe inhibition of ISV sprouting and DLAV formation was also apparent at $48 \mathrm{hpf}$ based on kdrl: GFP expression in vegfa MO; clec14a-/- embryos compared to single clec14a mutants and vegfaa MO embryos (Fig. 5e-h). Thus, inhibition of Clec14a and Vegfaa function results in a synergistic interaction.

\section{Discussion}

Our results argue that clec14a participates in both vasculogenesis and angiogenesis in the zebrafish model system. clec14a mutant embryos display mild defects in angiogenesis. The defects are significantly more severe upon simultaneous depletion of $c 1 q r$, suggesting functional redundancy between the two genes. It was previously demonstrated that genetic mutants frequently exhibit upregulation of functionally homologous genes resulting in functional compensation [8]. Our results show that clqr expression is upregulated in clec14a mutants. It is likely that clqr can partially compensate for the absence of clec14a function, and therefore strong 


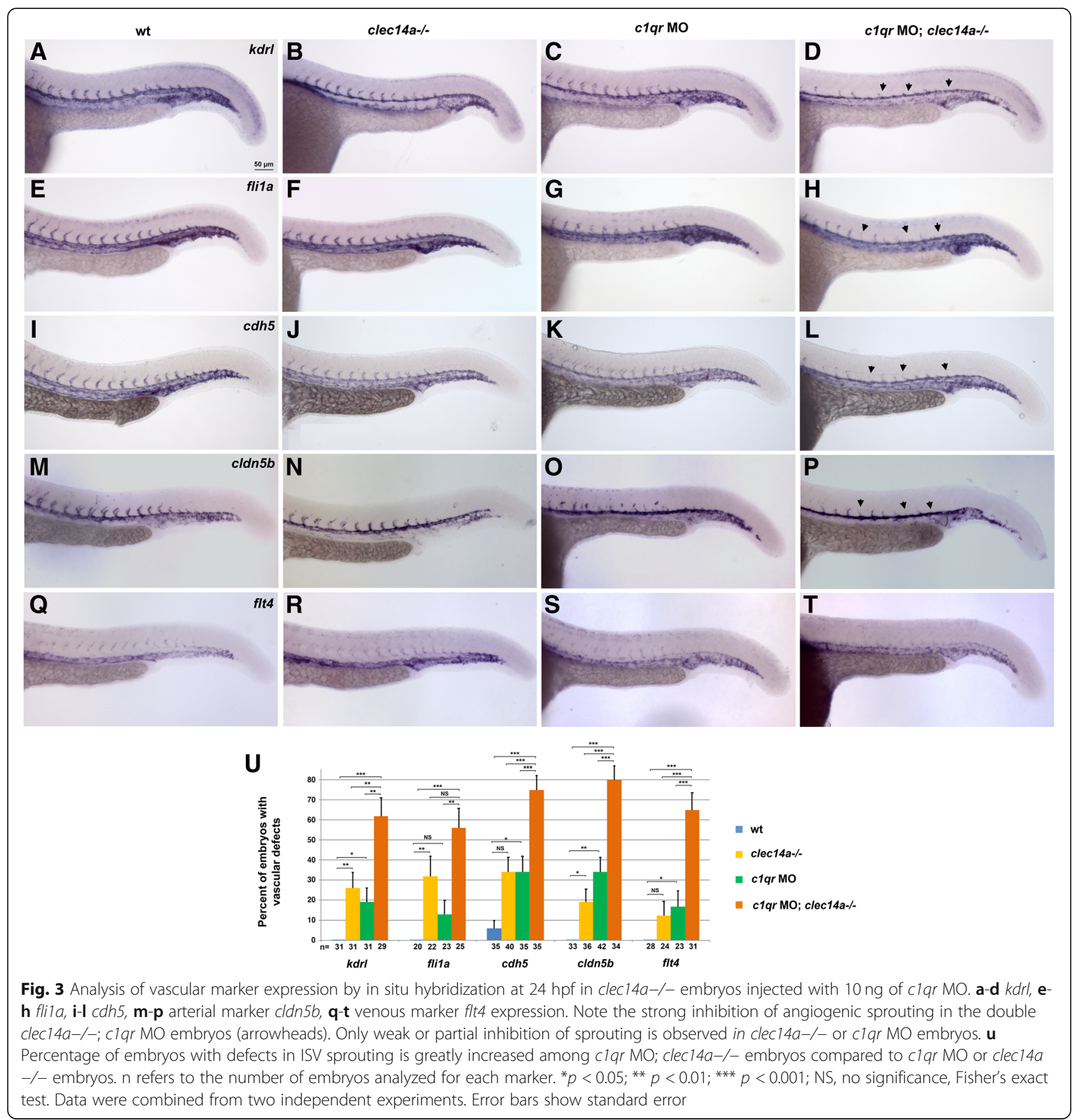

vascular defects are observed only when both homologs are inhibited. Similar redundant function between clec14a and c1qr in zebrafish embryos has been previously reported [5]. Differently from the previous study, we did not observe reduced survival among clec14a mutants. While the nature of $\operatorname{clec} 14 a^{\text {cils }}$ allele is different from the previously described c1qrl / clec14a ${ }^{c q 30}$ allele [5], clec14a $a^{\text {ci15 }}$ mutants show great reduction in clec14a mRNA expression likely due to the nonsense-mediated RNA decay, and therefore it is likely to be a null or close to null allele.
It is not clear why mouse and zebrafish clec14a mutants show quite different phenotypes. It is worth noting that previous studies have reported reduced angiogenesis upon Clec14a knockdown in vitro [2, 4], which is similar to the zebrafish clec14a mutant phenotype. Increased angiogenesis in mice was observed at much later stages (E13.5) [6] than the phenotypic analysis in ISV sprouting that we performed in zebrafish. It is possible that distinct vascular beds are affected differently by the loss of Clec14a function. The defects in ISV sprouting are quite mild, and are only pronounced upon combinatorial 

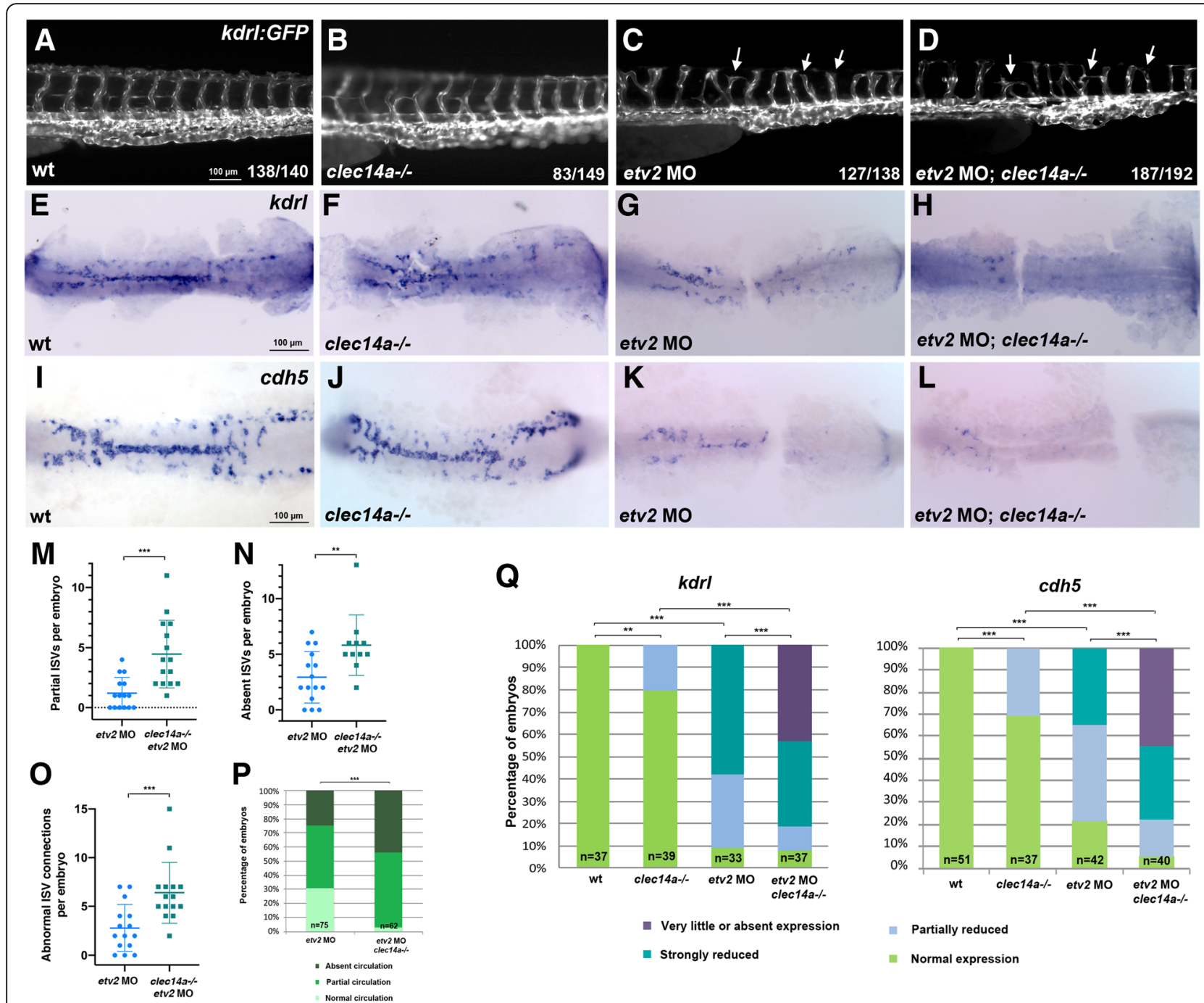

Fig. 4 Combinatorial interaction between etv2 and clec14a. a-d ISV sprouting defects (arrowheads) are increased in clec14a mutants injected with the low dose of etv2 MO2 (0.25 ng) compared with etv2 MO injection in wild-type embryos. kdrl: GFP transgenic embryos were imaged at 48 hpf; the trunk region is shown. e-I ISH analysis for $\mathrm{kdrl}(\mathbf{e}-\mathbf{h})$ and $c d h 5$ expression at the 15-16-somite stages. Flat-mounted embryos; only the trunk and tail region is shown. Note a significant reduction in cdh5 and kdrl expression in the embryos injected with low dose $(0.125 \mathrm{ng})$ of etv2 MO2. Much greater reduction is observed in etv2 MO; clec14a-/- embryos. m-o The number of partial (m) or absent (n) ISVs per embryo and abnormal ISV connections per embryo (o) in wild-type kdrl: GFP or clec14a-/-; kdrl: GFP embryos at $48 \mathrm{hpf}$ which were injected with $0.25 \mathrm{ng}$ of etv2 MO. 12-15 embryos were analyzed for ISV defects. ${ }^{* *} p<0.001$, t-Student's test. p Percentage of embryos with affected blood circulation at 48 hpf. All wild-type control and clec14a-/- embryos had normal blood circulation (not shown). To calculate statistical significance, embryos were compared with normal or defective circulation (combined partial and absent circulation categories) using Fischer's exact test, ${ }^{* * *} p<0.001$. q Percentage of embryos with normal or reduced $k d r l$ and $c d h 5$ expression. Statistical significance was calculated for wild-type versus clec14a-/and etv2 MO embryos using normal and reduced expression categories (all categories with reduced expression were combined), and for etv2 MO; clec14a-/- embryos versus clec14a-/- and etv2 MO embryos using very little or absent expression categories (normal, partially and strongly reduced expression categories were combined). ${ }^{* *} p<0.01,{ }^{* * *} p<0.001$, Fisher's exact test. Data were combined from two independent experiments

knockdown of clec14a and other genes involved in ISV sprouting. It is possible that Clec14a in mouse may also have an earlier role in promoting angiogenesis which is not apparent due to functional redundancy with C1qr or other related proteins.

Zebrafish clec14a expression was greatly downregulated or absent in cloche/npas $4 l$ or etv 2 mutants prior to
24 hpf [3, 9], while etv2 expression was not affected in clec14a/c1qrl ${ }^{c q 30}$ mutants [5], suggesting that clec14a functions downstream of npas $4 l$ and etv2. Intriguingly, clec14a mutants showed synergistic interaction with etv2 during vasculogenesis. The combinatorial loss of clec14a and partial knockdown of etv2 function resulted in a much greater reduction in vascular $k d r l$ and $c d h 5$ 


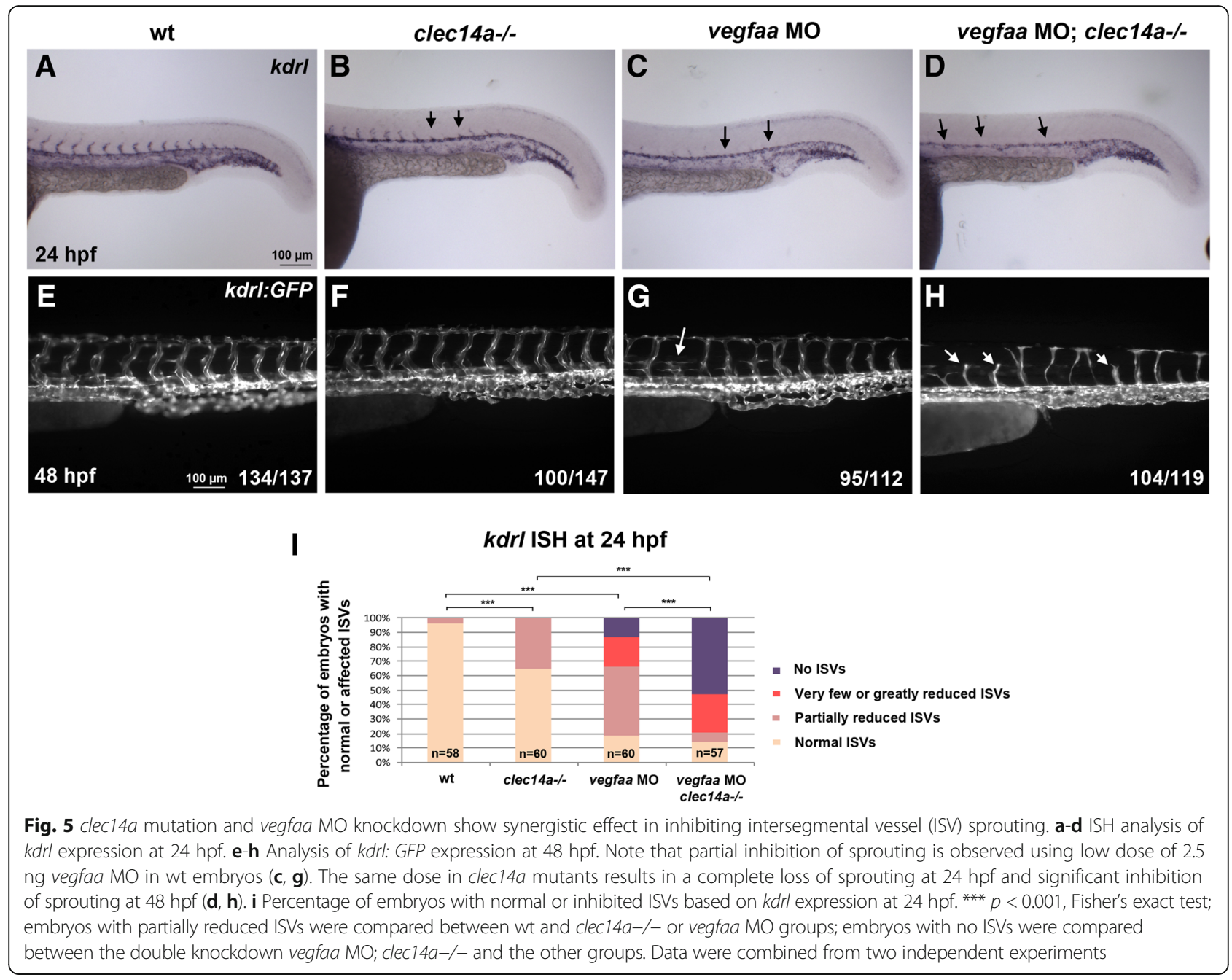

expression than separate mutation or knockdown of clec14a and etv2. Synergistic interaction observed under partial etv2 knockdown suggests that either clec14a functions downstream of etv 2 in the same pathway, or both clec14a and etv2 function in two parallel and converging pathways during vasculogenesis. Yet despite the major defects in vasculogenesis at mid-somitogenesis stages, the same clec14a-/-; etv2 MO knockdown embryos displayed relatively mild ISV sprouting defects at later stages. This partial recovery of vascular defects is likely due to the compensation of Etv2 deficiency by other ETS transcription factors such as Fli1b; we have previously demonstrated that Fli1b plays a major role in the partial recovery of defects in vasculogenesis observed in etv2 mutant embryos [12].

Vegf signaling plays multiple roles during vasculogenesis and angiogenesis [13]. It has been recently shown that Clec14a physically interacts with Vegfr3 in vitro, and that mouse Clec14a knockout embryos show reduced Vegfr3 expression and increased expression in Vegfr 2 which correlates with increased angiogenesis [6]. However, no increased angiogenesis was observed in zebrafish clec14a mutants. flt4/vegfr3 and $k d r l$ expression in the axial vasculature was not significantly affected in clec14a-/-; c1qr $\mathrm{MO}$ embryos, while ISV sprouting was reduced. It is possible that inhibition of angiogenesis may be a consequence of reduced Vegf signaling in clec14a mutants. Because Vegf signaling potentiates etv2 expression during vasculogenesis [14], this would also explain synergistic interaction between the etv2 and clec14a knockdown / mutation during vasculogenesis. However, synergistic interaction between clec14a mutation and vegfaa MO knockdown does not exclude a possibility that clec14a may also function in a separate parallel signaling pathway which promotes angiogenesis. Multiple other pathways including Angiopoietin-Tie2 and Delta-Notch signaling have been implicated in angiogenesis [15]. Further research will be required to test a potential clec14a involvement in these pathways.

\section{Conclusions}

This study demonstrates the requirement for clec14a in both vasculogenesis and angiogenesis in the zebrafish model system. clec14a functions partially redundantly 
with a related protein $\mathrm{C} 1 \mathrm{qr}$ / Cd93 during sprouting angiogenesis. In addition, clec14a genetically interacts with the ETS transcription factor etv2 during vasculogenesis, demonstrating its novel role in promoting differentiation of vascular endothelial progenitors. Furthermore, our results show synergistic genetic interaction between clec14a and Vegf signaling. These results will promote our understanding of the mechanisms that guide vascular development.

\section{Methods}

\section{Fish lines}

clec14a $a^{\text {cils }}$ line was obtained by TALEN mutagenesis. TALENs were designed to the single clec14a exon using the TAL Effector Nucleotide Targeter software at https://talent.cac.cornell.edu [16]. The target site TGTTCACTT GAACAAAAACTcattcacagatgcgcAGAATTATTGCAAA CCTGGA was chosen near the $5^{\prime}$ end of the exon. The spacer sequence indicated in lowercase contains the HhaI enzyme recognition site CGCG. TALEN constructs were generated using Golden Gate assembly [17]. mRNA for the left and right TALEN arms was synthesized using T3 mMessage mMachine Kit (ThermoFisher). $50 \mathrm{pg}$ each mRNA of was injected into 1-cell stage embryos. The following PCR primers flanking the TALEN recognition site were used to test for TALEN efficiency in pools of injected embryos: clec14a_F 5'-GCAGACATGGATTTCTGGA TGG-3', clec14a_R 5'-AGTGCTGTTGTCCACCGTC-3'. These primers amplify a $315 \mathrm{bp}$ product that was fully digested by HhaI to produce $116 \mathrm{bp}$ and $199 \mathrm{bp}$ products in uninjected embryos. Retention of the $318 \mathrm{bp}$ product in injected embryos indicated efficient TALEN mutagenesis. Adult carriers were identified by PCR genotyping using the same primers and HhaI enzyme digest. PCR products were sequenced using the clec14a_F primer to determine the deleted region.

Homozygous clec14 $4^{\text {ci15 }}$ embryos in $T g$ (kdrl: GFP) $)^{s 843}$ [18] background and control Tg (kdrl: GFP) ${ }^{s 843}$ embryos were obtained from incrosses of homozygous clec14 $14^{\text {ci15 }}$; $k d r l$ : GFP and wild-type $k d r l: G F P$ adults, respectively. Embryos were raised at $28.5^{\circ} \mathrm{C}$ or $32^{\circ} \mathrm{C}$ temperature. Embryonic staging was performed according to the established criteria [19].

\section{Phenotypic analysis}

Live embryos were analyzed at $28-72 \mathrm{hpf}$ stages for vascular defects based on kdrl: GFP expression. Embryos which contained any ISVs in the trunk and tail region which were either not fully extended, absent or formed abnormal connections were counted as embryos with vascular defects.

\section{Morpholinos}

A translation-blocking c1qr MO (GTCACTCTCATACT ACTCGCTTTAG, Gene-Tools Inc), a 5-base c1qr mismatch MO (GTCAgTCTgATAgTACTCggTTTAG), a standard control MO (CCTCTTACCTCAGTTACAA TTTATA, Gene-Tools Inc), a previously reported vegfaa MO (GTATCAAATAAACAACCAAGTTCAT) [11] and etv2 MO2 (CACTGAGTCCTTATTTCACTATATC) [9] were used for experiments. All injections were performed at 1-2-cell stage.

\section{In situ hybridization}

Whole mount in situ hybridization was performed using DIG-UTP labeled probes synthesized with T3, T7, or SP6 polymerase (Promega) as previously described [20]. Antisense RNA probes for the following genes were synthesized as previously described: $k d r l / f l k 1$ [21], fli1a [21], cdh5 [22], cldn5b [23], flt4 [21], clec14a / crl [3].

\section{CDNA analysis and qPCR}

RNA was purified from 15 to 20 wild-type and clec14a mutant embryos in kdrl: GFP background at $24 \mathrm{hpf}$ using PureLink RNA purification kit (Thermo Fischer Scientific). cDNA was synthesized using SuperScript VILO cDNA synthesis kit (Thermo Fischer Scientific). PCR product corresponding to the coding sequence of clec14a was amplified with the following primers: TAAG CACTCGAGCACCATGGATTTCTGGATGGTA TTACATC and TGCTTAAGATCTTTAGGTTTCCTC TTTTTCATTCACC. qPCR for $c 1 q r$ expression was performing using the following primers: GCTTGACTC AGTTACCTGACGG and TTTCTGCTCGCTGT CCAACCC. Amplification was performed using the SYBR green PCR master mix and Step One Plus real-time PCR system (Thermo Fischer Scientific). Quantification was normalized to ef1a expression which was amplified using the following primers: TCAC CCTGGGATGAAACAGC and ACTTGCAGGCCATG TGAGCAG. Two independent embryo replicates (15-20 embryos each) and 2-4 technical replicates for each sample were performed.

\section{Generation of c1qr:GFP reporter construct}

$939 \mathrm{bp} c 1$ qr upstream fragment was amplified by PCR from the CH211-202F3 BAC construct (obtained from Children's Hospital Oakland Research Institute) using Expand High Fidelity PCR System (Sigma-Aldrich) and the following primers: $c 1 q r-F$ : TCCATTTGCCTTCGGCTGGG and $c 1$ $q r-R:$ CGAGTAGTATGAGAGTGACGGG. GFP-polyA sequence was PCR amplified from XGM2 (Xenopus EF1aGFP-polyA construct) [24] using GFP-forward primer with an attached sequence that overlaps with the clqr promoter fragment, and SP6 24-mer primers: GCGAGTAGTATGAG AGTGACGGGATGAGTAAAGGAGAAGAACTTTTCAC TGG and CATACGATTTAGGTGACACTATAG. The final product was amplified by PCR using $c 1 q r-F$ and SP6 24-mer primers and the initial two PCR products as DNA templates. PCR product was gel-purified, diluted in $1 \mathrm{x}$ 
Danieau buffer $(58 \mathrm{mM} \quad \mathrm{NaCl}, \quad 0.7 \mathrm{mM} \quad \mathrm{KCl}, \quad 0.4 \mathrm{mM}$ MgSO4, $0.6 \mathrm{mM} \mathrm{Ca}(\mathrm{NO} 3)_{2}, 5 \mathrm{mM}$ HEPES, pH 7.6) and injected into the embryo blastomere at 1-cell stage at a dose of $50 \mathrm{pg}$ per embryo.

\section{Microscopy}

Embryos were whole-mounted in $2 \%$ methylcellulose on glass slides. Images were captured using a 10x / NA 0.3 objective on an AxioImager Z1 (Zeiss) compound microscope with an Axiocam ICC3 color camera or Axiocam MMR grayscale camera (Zeiss). Images in multiple focal plans were captured individually and combined using the Extended Focus module within the Axiovision software (Zeiss). For ISH images at mid-somitogenesis stages, embryos were deyolked and flat-mounted in the araldite medium (EM Sciences).

\section{Additional files}

Additional file 1 : Figure S1. clar MO inhibits reporter clar:GFP expression. (A) A diagram of the clar reporter construct. clar sequence of $939 \mathrm{bp}$ immediately upstream of the initiating ATG codon which contains the clar MO binding site was fused with the GFP-polyA sequence using the fusion PCR approach. $(B, C)$ GFP fluorescence in zebrafish embryos at the 22-24-somite stages which were injected with $50 \mathrm{ng}$ of c1 gr:GFP PCR product alone (B) or in combination with $10 \mathrm{ng}$ of clar $\mathrm{MO}$ (C). Trunk and tail region is shown. Note that the distribution of injected DNA is typically highly mosaic. $11 \%$ of embryos injected with c1 qr:GFP DNA showed multiple GFP+ cells in the tail region, while none of clar:GFP and clar MO co-injected embryos showed such expression $(p=0.01$, Fischer's exact test). Data were combined from two independent experiments. Figure S2. Injection of control MO (10 ng) does not enhance blood vessel defects in clec14a mutant embryos. (A-D) kdrl: GFP expression analysis at $48 \mathrm{hpf}$. (E) Percentage of embryos with vascular defects at 48 hpf. ${ }^{* * *}, p<0.001$; NS, not significant, Fischer's exact test. Data were combined from two independent experiments. Error bars show standard error. Figure S3. Injection of 10 ng of a 5 base-pair c1ar mismatch $\mathrm{MO}$ does not cause additional vascular defects in wild-type or clec 14a mutant embryos. (A-D) kdrl: GFP expression analysis at $48 \mathrm{hpf}$. (E) Percentage of embryos with vascular defects at 48 hpf. ${ }^{* *}, p<0.001$; NS, not significant, Fischer's exact test. Data were combined from two independent experiments. Error bars show standard error. (PDF 4910 kb)

Additional file 2: Movie S1. Blood circulation in wild-type embryos injected with low dose of etv2 $\mathrm{MO}(0.25 \mathrm{ng})$. Tail region is shown at 48 hpf, anterior is to the right. (MPG $14006 \mathrm{~kb}$ )

Additional file 3: Movie S2. Absent blood circulation in clec14a-/embryos injected with low dose of etv2 $\mathrm{MO}(0.25 \mathrm{ng})$. Tail region is shown at $48 \mathrm{hpf}$, anterior is to the right. (MPG $9052 \mathrm{~kb}$ )

\section{Acknowledgements}

We thank Sarah A. Mowery for her assistance with experiments during manuscript revision.

\section{Funding}

This research was supported by the National Institutes of Health award R01 HL107369 to S.S. and Cincinnati Children's Research Foundation Trustee Award to J.A.S. The funding bodies had no role in the design of the study and collection, analysis, and interpretation of data or in writing the manuscript.

\section{Availability of data and materials}

All data generated or analysed during this study are available from the corresponding author on reasonable request.

\section{Authors' contributions}

KP performed the experiments and analyzed the data, JAS generated clec $14 a$ mutant line, and edited the manuscript, SS designed and supervised the study, contributed to the analysis of the results, and wrote the manuscript. All authors read and approved the final manuscript.

\section{Ethics approval and consent to participate}

No human subjects were used in the study. Zebrafish embryo experiments were performed under animal protocol IACUC2016-0039, approved by the Instutional Animal Care and Use Committee at the Cincinnati Children's Hospital Medical Center.

\section{Consent for publication}

Not applicable.

\section{Competing interests}

The authors declare that they have no competing interests.

\section{Publisher's Note}

Springer Nature remains neutral with regard to jurisdictional claims in published maps and institutional affiliations.

\section{Author details}

'Division of Developmental Biology, Cincinnati Children's Hospital Medical Center, 3333 Burnet Ave, Cincinnati, OH 45229, USA. ²Present Address: Vilnius University Life Sciences Center, Sauletekio 7, 10223 Vilnius, Lithuania.

${ }^{3}$ Department of Pediatrics, University of Cincinnati College of Medicine, 3333 Burnet Ave, Cincinnati, OH 45229, USA.

Received: 6 June 2018 Accepted: 21 March 2019

Published online: 05 April 2019

\section{References}

1. Risau W. Differentiation of endothelium. FASEB J. 1995;9(10):926-33.

2. Mura M, Swain RK, Zhuang X, Vorschmitt H, Reynolds G, Durant S, Beesley JF, Herbert JM, Sheldon H, Andre M, et al. Identification and angiogenic role of the novel tumor endothelial marker CLEC14A. Oncogene. 2012;31(3):293305.

3. Sumanas S, Jorniak T, Lin S. Identification of novel vascular endothelialspecific genes by the microarray analysis of the zebrafish cloche mutants. Blood. 2005:106(2):534-41.

4. Rho SS, Choi HJ, Min JK, Lee HW, Park H, Park H, Kim YM, Kwon YG. Clec14a is specifically expressed in endothelial cells and mediates cell to cell adhesion. Biochem Biophys Res Commun. 2011;404(1):103-8.

5. Du J, Yang Q, Luo L, Yang D. C1 gr and C1 grl redundantly regulate angiogenesis in zebrafish through controlling endothelial Cdh5. Biochem Biophys Res Commun. 2017:483(1):482-7.

6. Lee S, Rho SS, Park H, Park JA, Kim J, Lee IK, Koh GY, Mochizuki N, Kim YM, Kwon YG. Carbohydrate-binding protein CLEC14A regulates VEGFR-2- and VEGFR-3-dependent signals during angiogenesis and lymphangiogenesis. J Clin Invest. 2017:127(2):457-71.

7. Bedell VM, Wang Y, Campbell JM, Poshusta TL, Starker CG, Krug RG 2nd, Tan W, Penheiter SG, Ma AC, Leung AY, et al. In vivo genome editing using a high-efficiency TALEN system. Nature. 2012:491(7422):114-8.

8. Rossi A, Kontarakis Z, Gerri C, Nolte H, Holper S, Kruger M, Stainier DY. Genetic compensation induced by deleterious mutations but not gene knockdowns. Nature. 2015;524(7564):230-3.

9. Sumanas $\mathrm{S}, \mathrm{Lin} \mathrm{S}$. Ets1-related protein is a key regulator of vasculogenesis in zebrafish. PLoS Biol. 2006;4(1):e10

10. Pham VN, Lawson ND, Mugford JW, Dye L, Castranova D, Lo B, Weinstein BM. Combinatorial function of ETS transcription factors in the developing vasculature. Dev Biol. 2007;303(2):772-83.

11. Nasevicius A, Larson J, Ekker SC. Distinct requirements for zebrafish angiogenesis revealed by a VEGF-A morphant. Yeast. 2000;17(4):294-301.

12. Craig MP, Grajevskaja V, Liao HK, Balciuniene J, Ekker SC, Park JS, Essner JJ, Balciunas D, Sumanas S. Etv2 and fli1b function together as key regulators of vasculogenesis and angiogenesis. Arterioscler Thromb Vasc Biol. 2015: 35(4):865-76.

13. Bautch VL VEGF-directed blood vessel patterning: from cells to organism Cold Spring Harb Perspect Med. 2012;2(9):a006452. 
14. Casie Chetty S, Rost MS, Enriquez JR, Schumacher JA, Baltrunaite K, Rossi A, Stainier DY, Sumanas S. Vegf signaling promotes vascular endothelial differentiation by modulating etv2 expression. Dev Biol. 2017;424(2):147-61.

15. Marcelo KL, Goldie LC, Hirschi KK. Regulation of endothelial cell differentiation and specification. Circ Res. 2013;112(9):1272-87.

16. Doyle EL, Booher NJ, Standage DS, Voytas DF, Brendel VP, Vandyk JK, Bogdanove AJ. TAL Effector-Nucleotide Targeter (TALE-NT) 2.0: tools for TAL effector design and target prediction. Nucleic Acids Res 2012;40:W117-22.

17. Cermak T, Doyle EL, Christian M, Wang L, Zhang Y, Schmidt C, Baller JA, Somia NV, Bogdanove AJ, Voytas DF. Efficient design and assembly of custom TALEN and other TAL effector-based constructs for DNA targeting. Nucleic Acids Res. 2011;39(12):e82.

18. Jin SW, Beis D, Mitchell T, Chen JN, Stainier DY. Cellular and molecular analyses of vascular tube and lumen formation in zebrafish. Development. 2005;132(23):5199-209.

19. Kimmel CB, Ballard WW, Kimmel SR, Ullmann B, Schilling TF. Stages of embryonic development of the zebrafish. Dev Dyn. 1995;203(3):253-310.

20. Jowett T. Analysis of protein and gene expression. Methods Cell Biol. 1999; 59:63-85.

21. Thompson MA, Ransom DG, Pratt SJ, MacLennan H, Kieran MW, Detrich HW 3rd, Vail B, Huber TL, Paw B, Brownlie AJ, et al. The cloche and spadetail genes differentially affect hematopoiesis and vasculogenesis. Dev Biol. 1998; 197(2):248-69

22. Larson JD, Wadman SA, Chen E, Kerley L, Clark K, Eide M, Lippert S, Nasevicius A, Ekker SC, Hackett PB, et al. Expression of VE-cadherin in zebrafish embryos: a new tool to evaluate vascular development. Dev Dyn. 2004;231(1):204-13.

23. Rost MS, Sumanas S. Hyaluronic acid receptor Stabilin-2 regulates Erk phosphorylation and arterial--venous differentiation in zebrafish. PLoS One. 2014:9(2):e88614.

24. Johnson AD, Krieg PA. pXeX, a vector for efficient expression of cloned sequences in Xenopus embryos. Gene. 1994;147(2):223-6.

Ready to submit your research? Choose BMC and benefit from:

- fast, convenient online submission

- thorough peer review by experienced researchers in your field

- rapid publication on acceptance

- support for research data, including large and complex data types

- gold Open Access which fosters wider collaboration and increased citations

- maximum visibility for your research: over $100 \mathrm{M}$ website views per year

At $\mathrm{BMC}$, research is always in progress.

Learn more biomedcentral.com/submissions 